\title{
Neural Coding of Tactile Texture: Comparison of Spatial and Temporal Mechanisms for Roughness Perception
}

\author{
Charles E. Connor and Kenneth O. Johnson \\ Phillip Bard Laboratories of Neurophysiology, Department of Neuroscience, The Johns Hopkins University School of \\ Medicine, Baltimore, Maryland 21205
}

\begin{abstract}
A previous study showed that roughness perception may depend on either temporal or spatial variations in firing rate among cutaneous mechanoreceptive afferents. The present study was designed to distinguish between these hypotheses. Plastic surfaces embossed with patterns of dots designed to produce predictable alterations in temporal and spatial firing rate variation were used as stimuli in psychophysical and neurophysiological experiments. Subjective roughness magnitudes obtained from psychophysical experiments fitted the predictions of the spatial but not the temporal hypothesis. In the neurophysiological experiments, the stimuli were scanned across the receptive fields of cutaneous mechanoreceptive afferents. Firing rate variation in the neural responses was measured using a range of temporal and spatial filters. Temporal variation was not correlated with roughness magnitude. Spatial variation, on a scale of 1-2 $\mathrm{mm}$ (one to two receptor spacings), was closely correlated with roughness.
\end{abstract}

Texture, an important aspect of visual, auditory, and tactile perception, comprises a number of subsidiary qualities, including roughness. Roughness has been studied psychophysically in all three modalities, especially in touch (e.g., Meenes and Zigler, 1923; Stevens and Harris, 1962; Lederman, 1982) and audition (c.g., Wendahl, 1966). In vision, roughness may be a derived approximation to the tactile sensation (cf. Tamura et al., 1978; Heller, 1982). The neural basis of roughness sensations has been investigated in the tactile system by performing combined psychophysical and neurophysiological experiments (LaMotte, 1977; Sathian et al., 1989; Connor et al., 1990). Recent evidence from such studies indicates that roughness, in contrast with other magnitude sensations (e.g., Johnson et al., 1973; Johnson, 1974; Delgutte, 1987; Winslow and Sachs, 1988), is not based on the mean firing rate of primary afferent neurons. Instead, subjective roughness appears to be related to the variations in firing rate produced when the skin is scanned across an uneven surface. Firing rate variation in slowly adapting (SA) mechanoreceptive

Received Oct. 3, 1991; revised Mar. 25, 1992; accepted Apr. 8, 1992.

This study was supported by NIH Grant R01 NS18787 and by the W. M. Keck Foundation. We thank James Vaccaro, who produced the stimulus surfaces used in this study.

Correspondence should be addressed to Kenneth $O$. Johnson, Department of Neuroscience, The Johns Hopkins University School of Medicine, 725 North Wolfe Street, Baltimore, MD 21205.

a Present address: Division of Biology, California Institute of Technology, Pasadena, CA 91125 .

Copyright (C) 1992 Society for Neuroscience $0270-6474 / 92 / 123414-13 \$ 05.00 / 0$ afferents is closely correlated with psychophysical estimates of roughness magnitude (Connor et al., 1990).

It remains unclear, however, whether the relevant variations are temporal or spatial. One possibility is that roughness is based on temporal fluctuations in firing rates of individual afferents. Such fluctuations are produced because receptive fields are stimulated intermittently as the skin passes across the raised elements of a rough surface. The variation in response rate across time transmits information about the unevenness of the surface: the size and sharpness of the raised elements are reflected in the amplitude of variation, and the spacing between elements is reflected in the period of variation. This type of information could be extracted by a central mechanism of the sort pictured in Figure $1 A$. The central neuron illustrated in Figure $1 A$ receives excitatory and inhibitory input from the same peripheral afferent, but the two inputs have different time delays. The central neuron does not respond to a constant input from the peripheral afferent because the excitatory and inhibitory effects are contemporaneous and cancel out. The central neuron does, however, respond to a time-varying input because, in this case, the excitatory effect due to an input peak can take effect before the corresponding inhibitory effect reaches the neuron. Thus, the central neuron is selectively responsive to temporal variation in the peripheral response. The strength of the central response would be determined by the amplitude of input variation and the degree of match between the period of input variation and the excitatory-inhibitory delay difference. The summed impulse rate across a population of such central neurons would reflect the overall amount of within-afferent temporal variation, and thus the roughness of the stimulus surface. The mechanism illustrated in Figure $1 A$ is a simplified version of the temporal mechanisms tested in this study.

Another possibility is that roughness depends on local spatial variations in firing rate between afferents arrayed across the skin surface. Such variations are due to the irregular pattern of skin indentation produced by a rough surface. The resulting twodimensional (2-D) pattern of firing rates across the afferent population transmits information about the unevenness of the surface. As in the temporal case, the size and sharpness of the raised elements affect the amplitude of spatial variation, and the spacing between elements affects the period of spatial variation. This information could be extracted by a central mechanism like that shown in Figure $1 B$. The central unit receives excitatory input from one peripheral afferent and inhibitory input from another afferent some distance away on the skin surface. A spatially uniform pattern of activity in the afferent population will not excite the central cell because the excitatory 
A. TEMPORAL MECHANISM

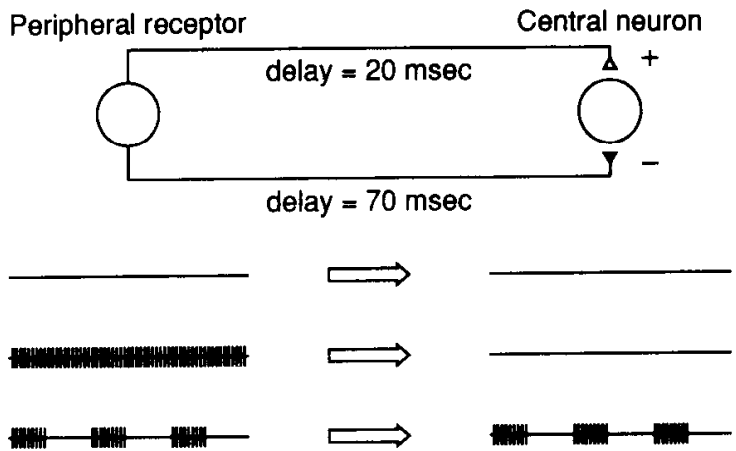

B. SPATIAL MECHANISM

Peripheral receptors
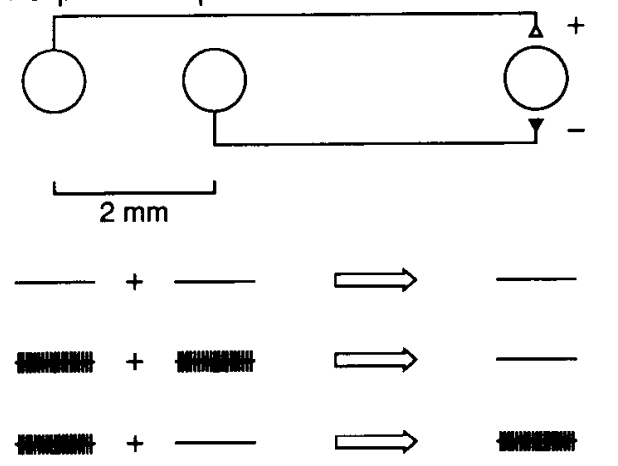

Figure 1. Mechanisms for roughness perception. $A$, Temporal mechanism that involves excitatory and inhibitory inputs from the same peripheral receptor but with different conduction delays. Peripheral firing patterns are represented on the left, and the resultant central firing patterns are represented on the right. Only intermittent activity in the peripheral receptor, such as that produced by scanning across a rough surface, leads to a response in the central neuron. $B$, Spatial mechanism that involves excitatory and inhibitory inputs from neighboring receptors. Only an irregular pattern of skin indentation and receptor activation, such as that produced by a rough surface, leads to a central response.

and inhibitory effects cancel. A patchy pattern of activity, however, will excite the central neuron whenever the excitatory afferent is stimulated and the inhibitory afferent is silent. The response rates of such central neurons will depend on the dif- ferences in firing ratc between stimulated and unstimulated afferents (i.e., the amplitude of local spatial variation) and on the distance between peaks and troughs of activity on the skin surface (i.e., the period of spatial variation; for the example shown, a peak to trough distance of $2 \mathrm{~mm}$ would produce the maximum effect). A population of such neurons could measure local spatial variation across the entire skin surface. The summed impulse rate of this population would reflect the overall roughness of the stimulus surface. The mechanism illustrated in Figure $1 B$ is a simplified version of the spatial mechanisms tested in this study.

Our previous studies failed to distinguish between temporal and spatial hypotheses because the stimuli (plastic surfaces containing embossed dots arranged in a square array) always produced parallel changes in temporal and spatial firing rate variation. For the present investigation, we designed stimuli that produce contrasting effects on temporal and spatial variation. As before, the stimuli were plastic surfaces containing embossed dots, but the dots were arranged in either horizontal rows or vertical columns (see Figs. 2, 3; horizontal corresponds to the direction in which the skin was scanned across the pattern). Spacing between dots along the rows and columns varied from 1.5 to $4.0 \mathrm{~mm}$ (center to center). It was predicted, on the basis of previous neurophysiological results, that spatial variation would increase as dot spacing increases in either direction (horizontal or vertical; see Fig. 3, solid circles). This is because closely spaced dots produce continuous bands of neural activity in the afferent population, and thus little spatial variation in the direction of close spacing. Dots spaced at about $4 \mathrm{~mm}$, in contrast, produce maximum variation in firing rate amplitude across the skin surface (Connor et al., 1990). Thus, spatial variation should be highest at the $4 \mathrm{~mm}$ dot spacing. Temporal variation should likewise increase as dot spacing increases in the horizontal or scanning direction (see Fig. 3, left panel, open circles). This is because close dot spacing in the scanning direction produces a relatively steady firing rate, while wider dot spacing in the scanning direction produces large fluctuations in firing rate. Temporal variation should, however, decrease as dot spacing increases in the vertical direction. For the closely spaced vertical stimuli, every afferent will be stimulated intermittently as its receptive field passes across the columns of dots and the empty spaces between. When every afferent is excited in this way, the summed measure of temporal variation across the population will be maximal. At wider vertical spacings, however, only some afferents will be stimulated in this way; others will be silent because their receptive fields are passing across empty rows.

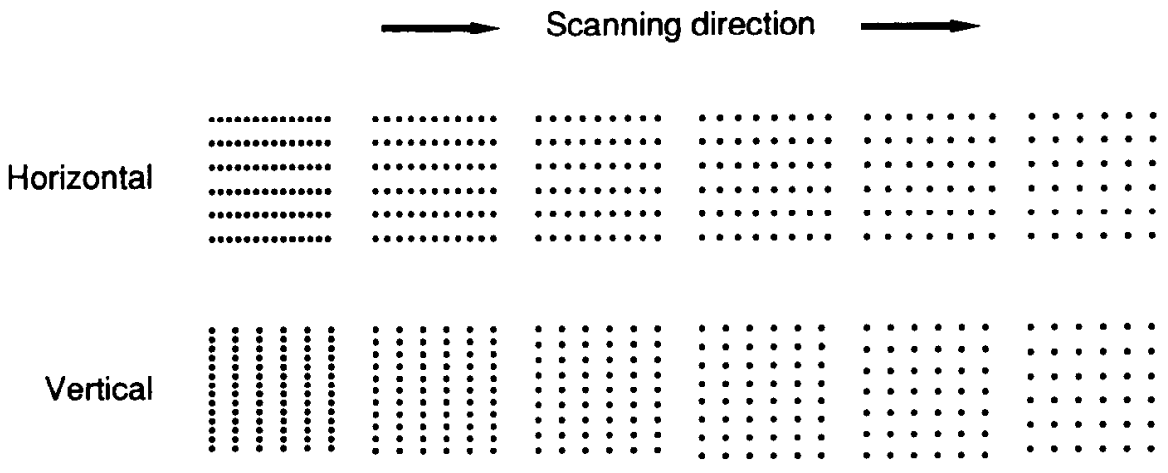

Dot spacing $(\mathrm{mm})$
2.0

2.5
Figure 2. Stimulus patterns. The stimuli were patterns of dots embossed on plastic surfaces. Each dot had the shape of a truncated cone $0.5 \mathrm{~mm}$ in height with a flat surface $0.5 \mathrm{~mm}$ in diameter. For the horizontal-row patterns, center-to-center spacing between dots varied from 1.5 to $4.0 \mathrm{~mm}$ in the horizontal direction and remained constant at $4.0 \mathrm{~mm}$ in the vertical direction. For the vertical-column patterns, spacing varied from 1.5 to $4.0 \mathrm{~mm}$ in the vertical direction and remained constant at $4.0 \mathrm{~mm}$ in the horizontal direction. The direction of motion relative to the skin surface is indicated. 


\section{$\leadsto$ Temporal hypothesis Spatial hypothesis}

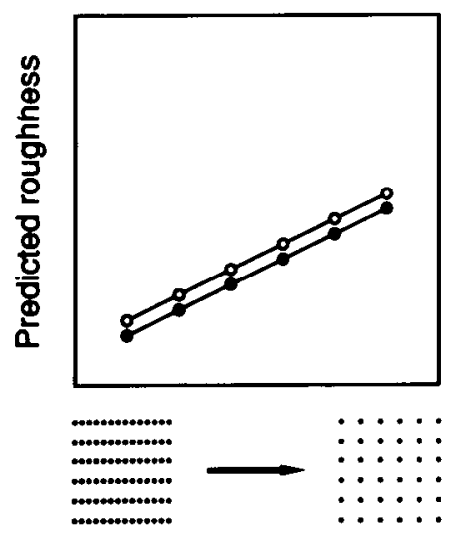

Horizontal
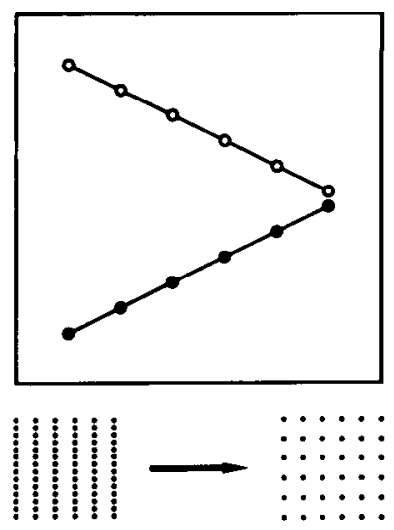

Vertical

Figure 3. Predicted roughness magnitude. The two panels show the expected behavior of roughness magnitude with respect to horizontal dot spacing (left) and vertical dot spacing (right), according to the temporal hypothesis (open symbols) and the spatial hypothesis (solid symbols). Dot spacing increases from left to right in both panels.

When part of the afferent population is silent, the summed measure of temporal variation is reduced.

Thus, the spatial and temporal hypotheses make different predictions about the relative roughness of these surfaces: surfaces with increased dot spacing in the vertical direction, as in Figure 3 (right panel), are expected to produce decreased temporal variation but increased spatial variation. In order to determine which hypothesis is correct, we performed psychophysical cxpcriments in which human subjects scanned their finger pads across the stimulus surfaces and reported roughness magnitude. The results followed the predictions of the spatial hypothesis. To obtain further confirmation, we performed neurophysiological experiments in which the stimuli were scanned across the receptive fields of monkey mechanoreceptive afferents. We determined the temporal and spatial firing rate variation evoked by the patterns, using analytical measures meant to approximate central mechanisms of the type discussed above. As a test of the two hypotheses, we examined the correlations between the temporal and spatial variation measures and the psychophysical reports of roughness magnitude.

\section{Materials and Methods}

Stimulus surfaces. The method for producing embossed plastic surfaces is described in Johnson and Phillips (1988). The surfaces used in this study contained dots with a relief height of $0.5 \mathrm{~mm}$. Each dot had the shape of a truncated cone with a flat top $(0.5 \mathrm{~mm}$ in diameter) and sides sloping out at an angle of $60^{\circ}$. Dots were arranged in horizontal rows or vertical columns (horizontal $=$ scanning direction). Spacing between the dots in one direction (either horizontal or vertical) varied from 1.5 to $4.0 \mathrm{~mm}$ in $0.5 \mathrm{~mm}$ increments. Spacing in the other direction was $4.0 \mathrm{~mm}$ (see Fig. 2). The stimulus surfaces for the neurophysiological experiments were arrayed in a strip $315 \mathrm{~mm}$ long and $35 \mathrm{~mm}$ wide that was wrapped around a 100-mm-diameter aluminum drum. Each stimulus occupied at least $25 \mathrm{~mm}$ in the longitudinal (scanning) direction. The stimulus surfaces for the psychophysical experiments were individual $50 \mathrm{~mm}$ squares mounted on flat Plexiglas acrylic blocks. These blocks were inserted by the experimenter into an apparatus designed to hold them firmly while the subject scanned the pad of his index finger across the surface.

Psychophysical methods. Subjects sat with the right arm resting on the surface of a table; the hand was pronated and screened from view

by a curtain. On each trial, the experimenter placed a different stimulus surface under the subject's hand, aligned so that the desired scanning direction was at right angles to the subject's forearm. The subject was instructed to palpate the stimulus with the distal pad of the forefinger, making back-and-forth scanning motions in the frontoparallel direction only. The subject was asked to report a numerical value proportional to the roughness of the stimulus, using any range of values that seemed appropriate. Before the experiment, a series of sample stimuli were presented so that the subject could choose a numerical scale. During the experiment, each of the 11 stimulus surfaces was presented five times in random order. The subjects were 36 medical students, 23 male and 13 female.

Neurophysiological methods. Experiments were conducted on anesthetized macaque monkeys (Macaca mulatta) weighing between 3.0 and $5.0 \mathrm{~kg}$. Single cutaneous mechanoreceptive afferent fibers were dissected from the median or ulnar nerves using methods described previously (Phillips and Johnson, 1981). Afferents were classified as slowly adapting (SA), rapidly adapting (RA), or pacinian ( $\mathrm{PC}$ ) on the basis of responses to a vibrating punctate probe (Talbot et al., 1968). Only SA and RA afferents with receptive fields on the distal pad of one of the digits (D2D5) were considered in this study. The stimulus surfaces, wrapped around the aluminum drum, were applied to the receptive fields using a rotating drum stimulus device described by Johnson and Phillips (1988). The stimuli were lowered onto the skin with a contact force of $30 \mathrm{gm}$ and rotated across the receptive fields at a speed of $50 \mathrm{~mm} / \mathrm{sec}$. These stimulus conditions approximate the pressure and speed used by human subjects in roughness estimation experiments (see Connor et al., 1990). Psychophysical studies by Lederman (1974) have shown that variations in force between 30 and $100 \mathrm{gm}$ and velocity between 10 and $250 \mathrm{~mm} /$ sec have virtually no effect on roughness judgments; moreover, neural responses to these patterns are relatively invariant over large differences in contact force and scanning velocity (Johnson and Lamb, 1981). After each full rotation of the stimulus pattern, the drum was shifted $0.2 \mathrm{~mm}$ in the axial direction (at right angles to the direction of rotation). This sequence was repeated at least 70 times, producing a total traverse of $14.0 \mathrm{~mm}$ in the direction orthogonal to rotation. The effect was to scan the receptive field across the entire group of stimulus patterns in a set of parallel sweeps. The occurrence times of action potentials evoked by the moving stimulus surface and position signals indicating the rotational and translational positions of the drum were collected.

Analysis. The psychophysical data were normalized by dividing each numerical response by the grand mean of all responses for the subject. Normalization was necessary because subjects were allowed to choose different numerical scales in reporting roughness. The normalized values were averaged across trials for each stimulus surface. Finally, the data were averaged across subjects to produce mean roughness judgments for each of the 11 stimuli.

Neurophysiological data were processed by correlating times of occurrence for action potentials with times of occurrence for stimulus position signals. The action potentials evoked by each sweep of a stimulus pattern across the receptive field were plotted as rows of event markers, and the rows stacked one on top of another to form a raster called a spatial event plot (SEP). The SEP effectively locates each action potential with respect to the part of the stimulus patlerin in contact with the finger at the time the action potential occurred. Apart from an unknown, constant error due to propagation delay between the receptors and the recording site, action potentials are located with a precision of approximately $10 \mu \mathrm{m}$ (Johnson and Phillips, 1988). The response of the neuron to any particular part of the pattern is thus represented by the action potentials plotted at the corresponding location in the SEP. The instantaneous firing rate in response to each part of the pattern was calculated by dividing the SEP into $0.2 \mathrm{~mm}$ hins and dividing the number of whole and fractional interspike intervals within each bin by the total dwell time in the bin ( $4 \mathrm{msec}$ at a scanning velocity of $50 \mathrm{~mm} /$ $\mathrm{sec}$ ). The resulting two-dimensional (2-D) array of firing rates was used in subsequent analyses of temporal and spatial firing rate variation.

Firing rate variation, whether temporal or spatial, was calculated by convolving the firing rate arrays with digital filters sensitive to local fluctuations in impulse rate. This procedure produced output arrays representing the amount of local variation at each $x-y$ point in the input firing rate arrays. The value at each point in the output array was computed as a weighted sum of input firing rates and thus had units of impulses per second (ips). The rectified average across each output array was taken as a measure of overall firing rate variation evoked by the respective stimulus pattern. The input arrays covered an integer number 
of dot spacings in the $\mathrm{x}$ and $\mathrm{y}$ directions yielding overall dimensions close to $15 \times 15 \mathrm{~mm}$.

Temporal variation was measured using one-dimensional (1-D) temporal Gabor filters based on the following formula:

$$
f(t)=\sin [2 \pi t / \lambda+\phi] * \exp \left[-t^{2} / 2 \sigma^{2}\right]
$$

where $t$ specifies temporal position, $\sigma$ is the standard deviation of the Gaussian distribution, $\lambda$ is the temporal period of the sinusoid, and $\phi$ is the phase of the sinusoid relative to $t=0$. All the results reported here were averaged across four values of $\phi(\phi=0, \pi / 2, \pi, 3 \pi / 2$ radians). The values for $\lambda$ and $\sigma$ were varied as described in Results. The resolution of the filter was the same as the resolution of the firing rate array, 4 msec.

Spatial firing rate variation was measured with 2-D spatial Gabor filters based on the following formula:

$$
f(x, y)=\sin [2 \pi(x \cdot \sin (\theta)-y \cdot \cos (\theta)) / \lambda+\phi] \cdot \exp \left[-\left(x^{2}+y^{2}\right) / 2 \sigma^{2}\right],
$$

where $x, y$ specifies spatial position, $\sigma$ is the standard deviation of the 2-D Gaussian, $\theta$ is the orientation of the spatial sinusoidal component of the filter (i.e., the orientation of the longitudinal positive and negative bands), $\lambda$ is the spatial period of the filter, and $\phi$ is the phase of the sinusoidal plane wave relative to the center. Results reported in this article were averaged across four values of $\phi(0, \pi / 2, \pi, 3 \pi / 2$ radians) and six values of $\theta\left(0-150^{\circ}\right.$ in $30^{\circ}$ increments). The values for $\lambda$ and $\sigma$ were varied systematically.

Spatial firing rate variation was also measured with 2-D difference of Gaussian (DOG) filters based on the following formula:

$$
f(x, y)=\frac{\exp \left[-\left(x^{2}+y^{2}\right) / 2 \sigma_{1}^{2}\right]}{2 \pi \cdot \sigma_{1}^{2}}-\frac{\exp \left[-\left(x^{2}+y^{2}\right) / 2 \sigma_{2}^{2}\right]}{2 \pi \cdot \sigma_{2}^{2}},
$$

where $x, y$ specifies spatial position and $\sigma_{1}$ and $\sigma_{2}$ are the standard deviations of the 2-D Gaussian distributions. Values for $\sigma_{1}$ and $\sigma_{2}$ were varied as described in Results. For any given pair of values, the results were averaged across two conditions, one in which $\sigma_{1}$ was less than $\sigma_{2}$ (producing an "on-center" filter, with a positive center and negative surround) and one in which the values for $\sigma_{1}$ and $\sigma_{2}$ were interchanged (producing an "off-center" filter, with negative center and positive surround).

Psychophysical and neurophysiological results were compared using statistical measures of correlation as well as by graphical inspection. Prior to comparison, psychophysical data were normalized and averaged across subjects, as described above. Neurophysiological measures of mean firing rate or firing rate variation were calculated separately for each fiber and then averaged across fibers. The correlation coefficients reported below are based directly on these averaged values. Correlations were not improved substantially by performing logarithmic transformations on one or both sets of values; that is, there was no suggestion of a nonlinear relationship between subjective magnitude and any of the measures tested.

\section{Results}

\section{Psychophysical results}

The stimuli used here were specifically designed to determine whether roughness is related to spatial or temporal variability in neural activity. If roughness sensations are based on spatial variation in cutaneous receptor responses across the skin surface, then perceived roughness magnitude should increase with larger dot spacings in both the horizontal (scanning) and vertical directions (see introductory remarks and Fig. 3). If, instead, roughness is based on temporal fluctuations in receptor activity, roughness magnitude should still increase with larger horizontal spacings but should decrease with larger vertical spacings. The results favored the spatial hypothesis: perceived roughness increased with dot spacing in both directions (see Fig. 4). Roughness magnitude was judged to be highest for those surfaces with dot spacings close to $4.0 \mathrm{~mm}$ and lowest for surfaces with dot spacings close to $1.5 \mathrm{~mm}$. Temporal variation, in contrast, is bound to be highest for vertical spacings close to $1.5 \mathrm{~mm}$ (see introductory remarks). These results indicate that roughness

\section{PSYCHOPHYSICAL RESULTS}

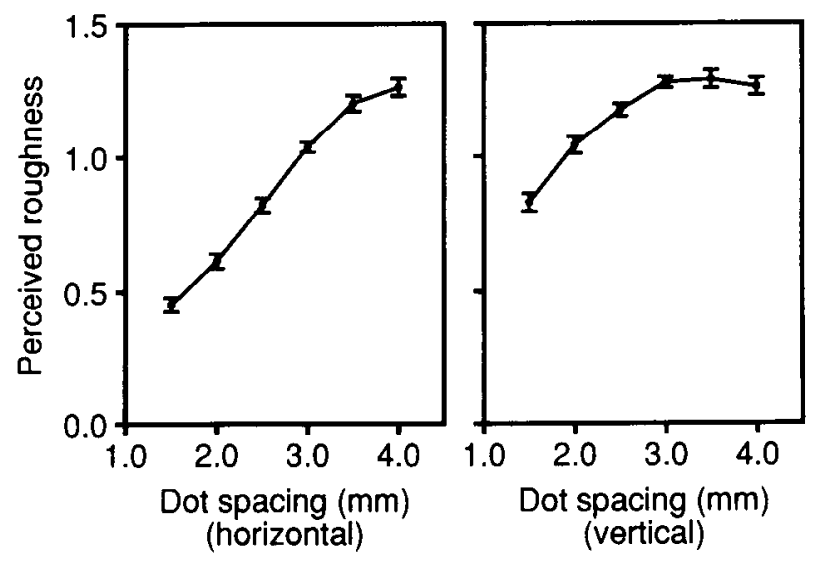

Figure 4. Psycophysical results. Roughness magnitude was averaged across 36 subjects after normalization within subjects to correct for differences in scale. The relationship of roughness to horizontal dot spacing is shown in the left panel; the relationship for vertical dot spacing is shown in the right panel. Error bars indicate SEM for each point.

sensations are based on temporal variation in cutaneous afferent activity.

Roughness was judged to be particularly low for closely spaced horizontal rows of dots (average normalized roughness $=0.45$ ). Much higher values were reported for closely spaced vertical columns (average normalized roughness $=0.83$ ). Any hypothetical neural mechanism must explain this difference between the horizontal and vertical stimuli, as well as the general decline in roughness at closer dot spacings.

\section{Neurophysiological results}

Neurophysiological data were collected from 14 SA and 16 RA afferents. Previous results had shown that roughness magnitude is closely correlated with firing rate variation in SA responses and somewhat correlated with variation in RA responses. PC afferents were not included in this study, because $\mathrm{PC}$ responses appear to be unrelated to roughness magnitude (Connor et al., 1990). The data were used to construct SEPs (see Materials and Methods) showing the neural responses to each portion of the 2-D stimulus surfaces. Sample sections from SEPs for each of the stimulus patterns are shown in Figure 5. As predicted, stimuli with dot spacings near $1.5 \mathrm{~mm}$ produced fairly continuous bands of activity. As dot spacing increased to $4.0 \mathrm{~mm}$, the activity resolved into separate peaks corresponding to individual dots.

The weakest activity was evoked by horizontal rows of dots spaced at $1.5 \mathrm{~mm}$. The reason for this weak activity is the close dot spacing in the scanning direction. As a result of this close spacing, stimulus motion produces relatively little dynamic modulation of skin indentation. The skin overlying the rows remains almost permanently indented, as if by a stationary grating. Under these conditions, the responses of peripheral afferents, even SAs, adapt significantly, producing low firing rates. Responses to vertical columns of dots were stronger because for these stimuli the spacing in the scanning direction is $4.0 \mathrm{~mm}$. As a result of this wide spacing, stimulus motion produces a repeating cycle of skin indentation and release, preventing adaptation and producing high transient firing rates. These differences in response strength are a likely explanation of the dis- 


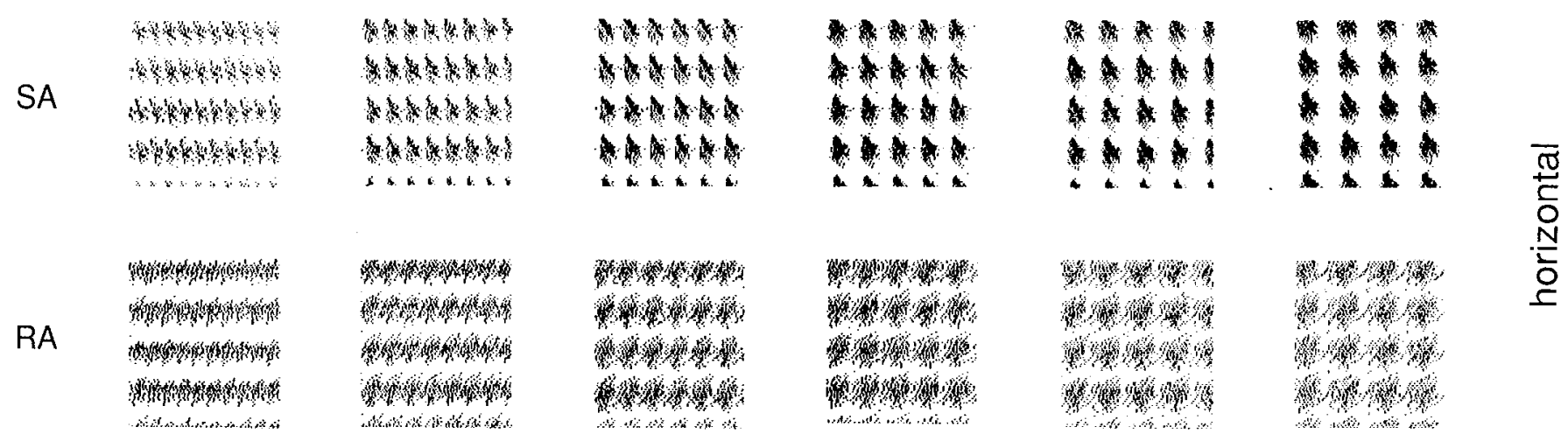
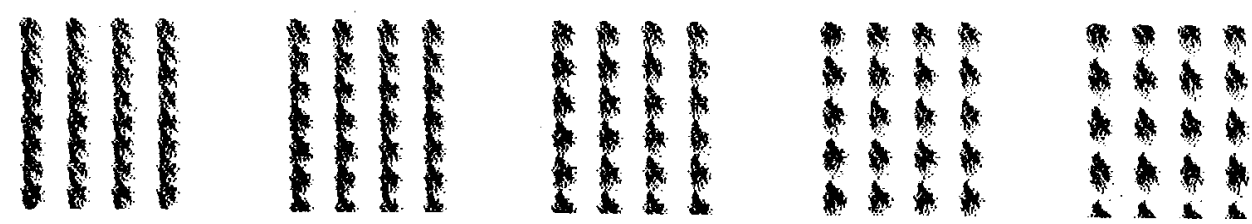

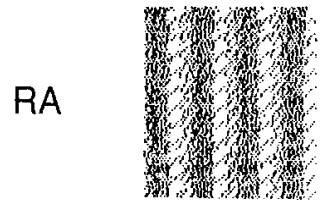

1.5

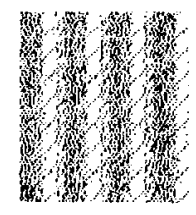

2.0

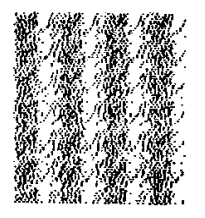

2.5

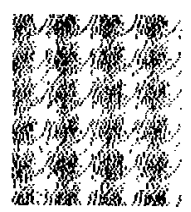

3.0

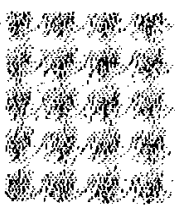

3.5
1) mols , 30,3

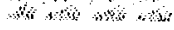

4.0

\section{Dot spacing $(\mathrm{mm})$}

Figure 5. Neural responses. Sample sections of SEPs for SA and RA fibers showing responses to horizontal (top) and vertical (bottom) dot patterns. Each tick mark represents the occurrence of an action potential. Each line of ticks represents the responses to a single sweep of the stimulus across the receptive field. Successive lines were recorded after successive $0.2 \mathrm{~mm}$ shifts of the stimulus (perpendicular to the scanning direction).

crepancy between perceived roughness of the horizontal and vertical stimulus patterns. The horizontal patterns produce a weaker response at all dot spacings (excepting $4.0 \mathrm{~mm}$, where the vertical and horizontal dimensions are equal) and thus less variation in neural activity. Therefore, both the temporal and spatial hypotheses would predict that the horizontal patterns should evoke a weaker sensation of roughness.

\section{Analysis}

In the previous study, hypothetical central mechanisms based on mean firing rate, temporal variation, and spatial variation in afferent responses were tested for their ability to explain perceived roughness magnitude (Connor et al., 1990). The same mechanisms were tested in the present study. This was done in order to (1) confirm the earlier finding that roughness magnitude is not based on mean firing rate, (2) confirm the conclusion drawn from the psychophysical data (see above) that roughness is not based on temporal variation, and (3) determine whether roughness can still be explained in terms of spatial variation.

Calculation of mean firing rate, temporal variation, and spatial variation was accomplished by initially transforming the data from each neuron into an SEP (see Materials and Methods and Fig. 5). A 2-D array of firing rate values was derived from the SEP (see Materials and Methods and Fig. 7). This 2-D array indicates the firing rate of the neuron in response to each part of the 2-D stimulus pattern. Mean firing rate was derived by simply averaging across the entire array. The resulting values were averaged across neurons to produce the final numbers used for comparison with the psychophysical data.

Temporal variation is represented in the SEP by variation in the horizontal or scanning direction. Each horizontal row in the SEP shows the response of the afferent fiber during one sweep across the stimulus pattern. Firing rate variation during each sweep was measured by convolving the horizontal row of firing rate values with a digital filter sensitive to local fluctuation. As an example, for the case of a filter 20 values wide, the 20 values in the filter were multiplied point for point with the first 20 values in the rate array. The resulting 20 products were summed to give the first value in the output array. This value represents the amount of variation across the first 20 values in the rate array, as measured by that particular filter. The filter was then "shifted" one position to the right and multiplied against values 2-21 in the input array, to obtain the second value in the output array. The filter was shifted repeatedly in the same fashion across each row in the SEP to produce the rest of the output array. The output array represents the response of the system across 

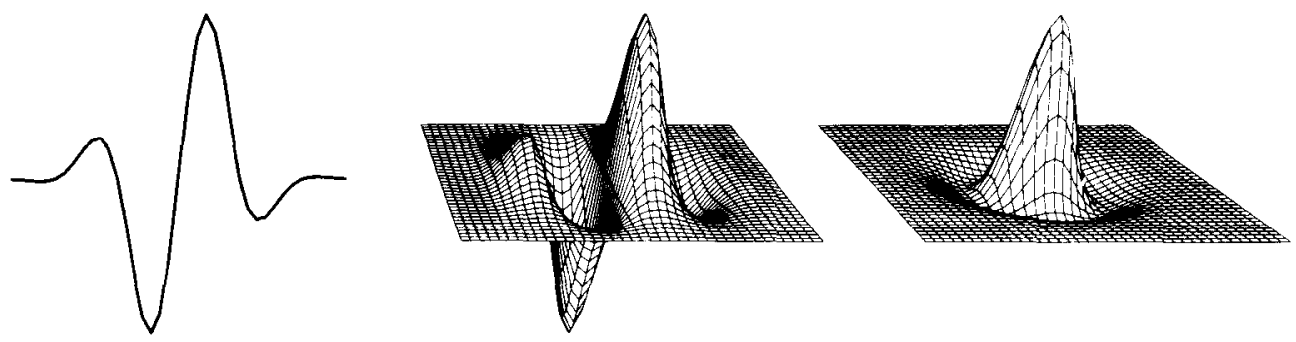

time. (Negative output values were replaced with zeros since firing rates cannot be negative.) Overall temporal variation is given by the average output across time.

The filters used here to measure temporal variation were 1-D Gabor filters (Gabor, 1946; see Materials and Methods). An example of such a filter is shown in Figure 6. Gabor filters consist of a sinusoidal function that grows and fades in amplitude within a Gaussian envelope. A Gabor filter provides a measure of localized, periodic variation. The specific kind of variation that is measured depends on the sinusoidal period, the SD of the Gaussian envelope, and the phase of the sinusoid relative to the location of the Gaussian mean. These three parameters completely define a 1-D Gabor filter. The filter shown in Figure 6
Figure 6. Temporal and spatial filters. The temporal Gabor filter shown here is based on a sinusoid with a period of $56 \mathrm{msec}$ modified by a Gaussian envelope with an SD of $22.4 \mathrm{msec}$ (resolution $=4 \mathrm{msec}$ ). The phase difference between the sinusoid and the Gaussian is 0 . The spatial Gabor filter is based on a sinusoidal plane wave with a period of $2.8 \mathrm{~mm}$ and a 2-D Gaussian with an $\mathrm{SD}$ of $1.12 \mathrm{~mm}$ (resolution $=$ $0.2 \mathrm{~mm}$, phase $=0$ ). The DOG filter represents the difference between a pair of 2-D Gaussians with SDs of $0.6 \mathrm{~mm}$ and $0.9 \mathrm{~mm}$. In this case, the narrower Gaussian was positive and the broader Gaussian was negative, so the filter has an "on-center" shape. is functionally similar to the simpler mechanism diagrammed in Figure $1 A$, with one large positive input and a delayed (more leftward) negative input. The negative lobe of the filter integrates activity from a slightly earlier period than the positive lobe. This corresponds to the longer delay on the inhibitory line in Figure $1 A$. The effect in both cases is that the system does not respond to a steady input but does respond to a time-varying input of the type produced when the peripheral receptor is scanned across a rough surface. The process of convolving a 1-D Gabor filter with an SEP is depictcd in the left panel of Figure 7.

Spatial variation is represented in the SEP by the amount of 2-D fluctuation in firing rate. The SEP can be taken to approx-

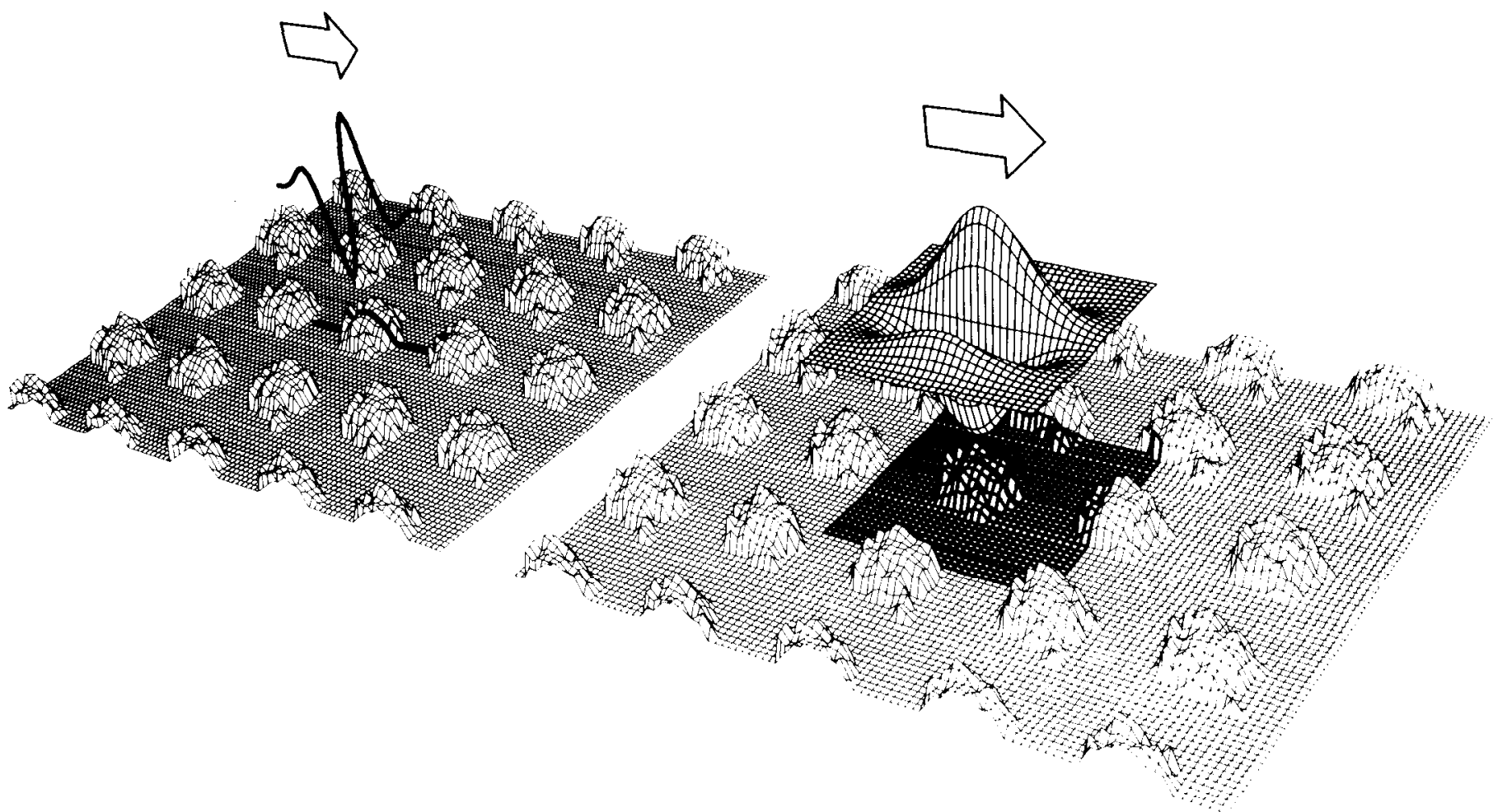

Figure 7. Convolution of temporal and spatial filters with neural data. These examples show the responses of an SA afferent to the $4.0 \times 4.0 \mathrm{~mm}$ stimulus. The SEP plot for the afferent (see Fig. 4) has been converted into a 2-D rate histogram. Rate is represented here as height; each hillock represents the response to one dot. The temporal and spatial filters are in effect superimposed on the neural data and corresponding points are multiplied. The resulting products are summed to give a single number. This process is repeated across all possible superpositions and the results averaged. The temporal filter measures rate variation only in the temporal direction (i.e., the scanning direction; see arrows). The spatial filter measures variation in the direction of the sinusoidal plane wave (in this case, perpendicular to the scanning direction). Spatial filter results are averaged across multiple orientations to produce a measure of 2-D variation. 


\section{A. SA MEAN RATE}

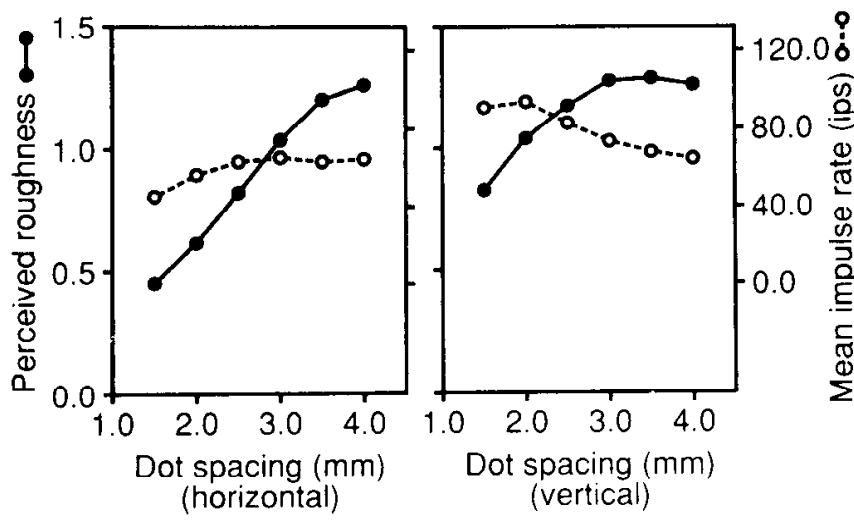

\section{B. RA MEAN RATE}
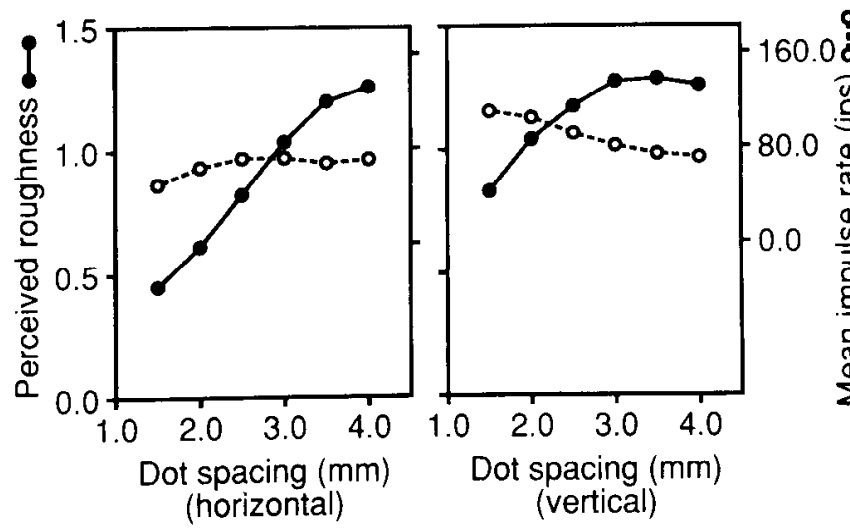

Figure 8. Mean rate and roughness magnitude versus dot spacing. The left hand ordinates represent normalized roughness magnitude, which is plotted with solid symbols. The right hand ordinates represent mean firing rate in impulses per second, plotted with open symbols. The two ordinates are scaled and translated relative to one another according to a linear regression between roughness and mean rate. $A$, SA mean rate; average $\mathrm{SEM}=7.1 \mathrm{ips}$; correlation coefficient $=0.404$. $B$, RA mean rate; average $\mathrm{SEM}=5.8 \mathrm{ips}$; correlation coefficient -0.301 .

imate the activity of an entire population of receptors arrayed in two dimensions across the skin surface (cf. Phillips et al., 1988). This assumption is justified by the close similarities in response properties between neurons within the separate populations (SA and RA). Because of these similarities, the response of the entire receptor array to a 2-D stimulus can be estimated from the response of a single receptor to all the various points in the 2-D stimulus. Thus, the SEP can be regarded as roughly equivalent to the $2-\mathrm{D}$ pattern of activity in the receptor population evoked by the stimulus at a given instant in time. Spatial variation in this $2-\mathrm{D}$ pattern was measured by convolving the firing rate array with a 2-D digital filter sensitive to local fluctuations in amplitude. The procedure is analogous to the measurement of temporal variation described above but extended to two dimensions. The 2-D filter is multiplied point for point with the $2-D$ patch of values in the upper left corner of the firing rate array. The products are summed to give the value in the upper left corner of the output array. The filter is shifted one position to the right to give the next value in the output array. Once this has been carried out for an entire row, the filter is shifted back to the left but one position down, and stepped across in the same fashion to produce the second row in the output array, and so forth. The output array represents the response, at a given instant in timc, of a 2-D array of central units receiving inputs from different locations on the skin surface. (Again, negative values are replaced with zeros since firing rates cannot be negative.) Overall spatial variation is given by the average across this array.

The filters used here to measure spatial variation were 2-D Gabor filters (Daugman, 1980, 1985; Marcelja, 1980; see Materials and Methods). An example is shown in Figure 6. Like its 1-D counterpart, a 2-D Gabor filter provides a measure of localized, periodic variation. The specific kind of variation that is measured depends on the frequency and orientation of the 2-D sinusoid, the spread of the Gaussian envelope, and the phase of the sinusoid relative to the envelope. Such filters have been used successfully to model simple receptive fields in visual cortex (e.g., Jones and Palmer, 1987; Worgotter and Holt, 1991). The filter shown in Figure 6 is functionally similar to the mechanism diagrammed in Figure $1 B$; both consist of a positive input paired with an adjacent negative input. The negative and positive lobes of the filter integrate inputs from neighboring locations on the skin surface. The system responds best to an input pattern of the kind produced when the skin is indented by a rough surface. The process of convolving a 2-D Gabor filter with an SEP is represented in the right panel of Figure 7.

Spatial variation was also measured with DOG filters. DOG filters are constructed by taking the difference between a pair of 2-D Gaussian functions of different sizes (see Materials and Methods). An example is shown in Figure 6. DOG functions have been used to model center-surround receptive fields in the visual system (Rodieck, 1965). DOG filters are useful in the present study for measuring spatial variation in multiple directions simultaneously. The spatial Gabor filters measure variation in only one direction (defined by the orientation of the sinusoidal plane wave); overall spatial variation is derived by averaging across orientations. A somewhat different result may be obtainable by using a filter that measures multidirectional variation in the first place. DOG filters accomplish this by virtue of the radial symmetry of their center-surround shape.

\section{Mean rate}

Mean firing rate was found to be poorly correlated with roughness magnitude, in agreement with previous results (Connor et al., 1990). The correlation between SA firing rate and roughness was 0.404 ; the correlation for RA firing rate was 0.301 . The relationship between mean rate and roughness is shown in Figure 8 . In this figure, the two $y$-axes are scaled and positioned relative to one another according to a linear regression between the psychophysical and neurophysiological values. This is done in order to produce maximum overlap between the roughness values (solid symbols) and the mean rate values (open symbols) so that the relationship between the two curves can be assessed visually. It is apparent that, whereas roughness increases with dot spacing, mean rate is relatively flat with respect to horizontal spacing and actually decreases with increasing vertical spacing. These results confirm the previous findings that roughness sensations are not based on mean firing rate.

\section{Temporal variation}

Temporal variation was measured using Gabor filters with various periodicities and temporal spreads. The temporal period of the filters (i.e., the wavelength of the sinusoid) was varied 
from 16 to $200 \mathrm{msec}$. Previous data suggested that a temporal period near $90 \mathrm{msec}$ would be optimal at a scanning velocity of $50 \mathrm{~mm} / \mathrm{sec}$ (Connor et al., 1990). The spread of the filters, as determined by the SD of the Gaussian envelope, was also varied. SD, which was specified in relation to the temporal period, ranged from 0.2 to 1.4 sinusoidal periods. This amounted to including from one-half to three cycles within the central three-fourths of the Gaussian envelope. The filter shown in Figure 6 has an SD of 0.4 periods and thus includes approximately one cycle. The phase of the sinusoid relative to the center of the Gaussian was set at $0, \pi / 2, \pi$, or $3 \pi / 2$ radians (phase $=$ 0 in Fig. 6). Altering the phase had no significant effect, so the results reported are based on averages across all four phases. Thus, each analysis of the neural data was based on a particular filter period and spread. The results were averaged across fibers and compared to the psychophysical data.

Temporal variation behaved as predicted in all cases. The best fit (highest correlation) between the psychophysical results and temporal variation was obtained with a Gabor filter with a period of $64 \mathrm{msec}$ and an SD (temporal spread) of $51 \mathrm{msec}$. The results obtained with these parameters are shown in Figure 9. As expected, temporal variation (open symbols) decreased as vertical dot spacing increased. This is in contrast to the psychophysical roughness judgments (solid symbols), which increased as vertical dot spacing increased. The apparent size of the decrease in temporal variation is small because the scale is compressed near the top of the graph to produce the maximum overlap. The actual percentage change is $34 \%$ for both SAs and RAs. The reason for the decrease in temporal variation is evident in the SEPs shown in Figure 5. At vertical dot spacings near $1.5 \mathrm{~mm}$, every scan across the pattern (i.e., every horizontal line in the SEP) fluctuates between high and low firing rates as the columns of dots pass across the receptive field. At vertical dot spacings near $4.0 \mathrm{~mm}$, this is true for only about half the temporal scans; the other half contain almost no activity. The SEPs reflect the fact that at $1.5 \mathrm{~mm}$ spacings every receptor will be intermittently stimulated, while at $4.0 \mathrm{~mm}$ spacings half the receptors will be silent, because their receptive fields are passing across the empty space between dots. Thus, overall temporal variation is bound to decrease as the dot spacing increases. The fact that roughness magnitude increases over the same range leads us to reject the hypothesis that roughness is based on within-afferent temporal variation in firing rate.

\section{Spatial variation}

Spatial variation was measured using 2-D Gabor filters of various periodicities and spreads. The spatial period of the filters (i.e., the wavelength of the sinusoidal plane wave) varied from 0.8 to $8.0 \mathrm{~mm}$. Data from the previous study suggested that a separation between the positive and negative peaks of the sinusoid of approximately $2 \mathrm{~mm}$ would be optimal; this corresponds to a spatial period near $4 \mathrm{~mm}$ (Connor et al., 1990). Filter spread, as determined by the SD of the Gaussian envelope, ranged from 0.2 to 1.4 spatial periods, which resulted in the inclusion of approximately one-half to three sinusoidal cycles within the filter. The phase of the sinusoid relative to the center of the Gaussian was set at $0, \pi / 2, \pi$, or $3 \pi / 2$ radians. As in the temporal analysis, changes in phase did not affect the results, so data for all four phases were averaged together. Filter orientation (i.e., direction of the sinusoid) was varied over a range of $0-150^{\circ}$ in $30^{\circ}$ increments. (Orientations of $180^{\circ}$ and above were redundant because of the chosen phase angles.) Orientation

\section{A. SA TEMPORAL VARIATION}

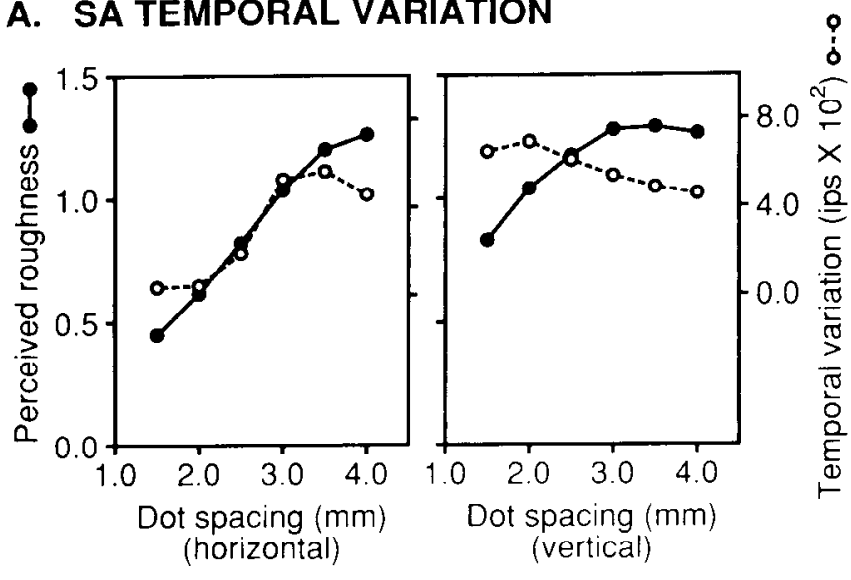

\section{B. RA TEMPORAL VARIATION}
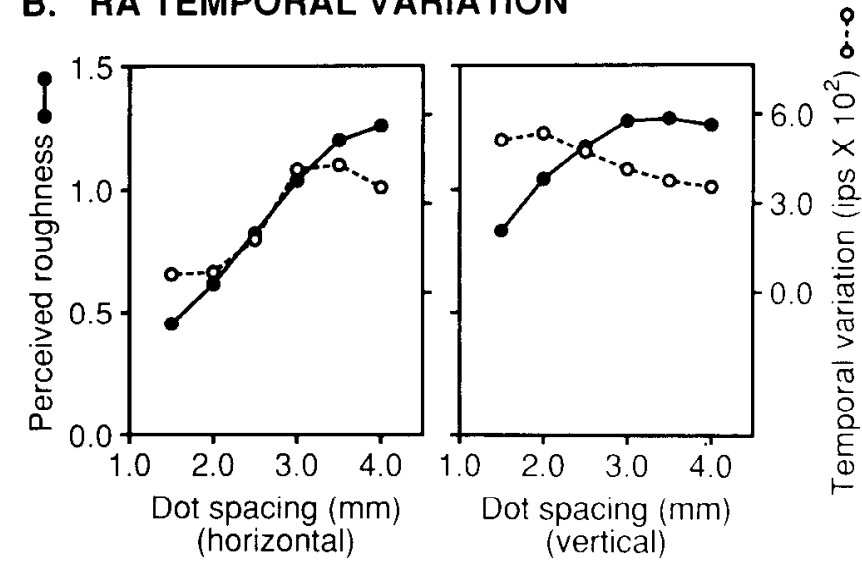

Figure 9. Temporal variation and roughness magnitude versus dot spacing, as in Figure 8. $A, \mathrm{SA}$ temporal variation; average $\mathrm{SEM}=52$ ips; correlation coefficient $=0.741 . B$, RA temporal variation; average $\mathrm{SEM}=27 \mathrm{ips}$; correlation coefficient $=0.714$.

had a substantial effect, but since the aim was to measure overall spatial variation (at a particular spatial frequency), the data were averaged across orientations. Thus, each analysis was based on a given combination of spatial period and filter spread, and the results were averaged across phase, orientation, and fibers before comparison with the psychophysical data.

As in our previous study, spatial variation in SA responses was closely related to roughness magnitude. The results obtained with optimal parameters $($ period $=2.8 \mathrm{~mm}, \mathrm{SD}=1.12 \mathrm{~mm}$ ) are presented in Figure 10. SA spatial variation mirrors both the decline in roughness at close dot spacings and the higher roughness values for the vertical stimuli. RA spatial variation behaves in a similar fashion but shows larger deviations from the psychophysical data. The simple, proportional relationship between SA spatial variation and roughness is evident in Figure 11 , where roughness magnitude is plotted directly against spatial variation. The correlation coefficient between SA spatial variation and roughness was 0.984 . The relationship between RA spatial variation and roughness magnitude is fitted reasonably well by a straight line (correlation coefficient $=0.938$ ) but the relationship is not proportional; that is, the intercept of a linear regression of roughness magnitude on RA spatial variation is not close to the origin. Moreover, because of the steep slope of the regression, surfaces that produce nearly identical values for 


\section{A. SA SPATIAL VARIATION}

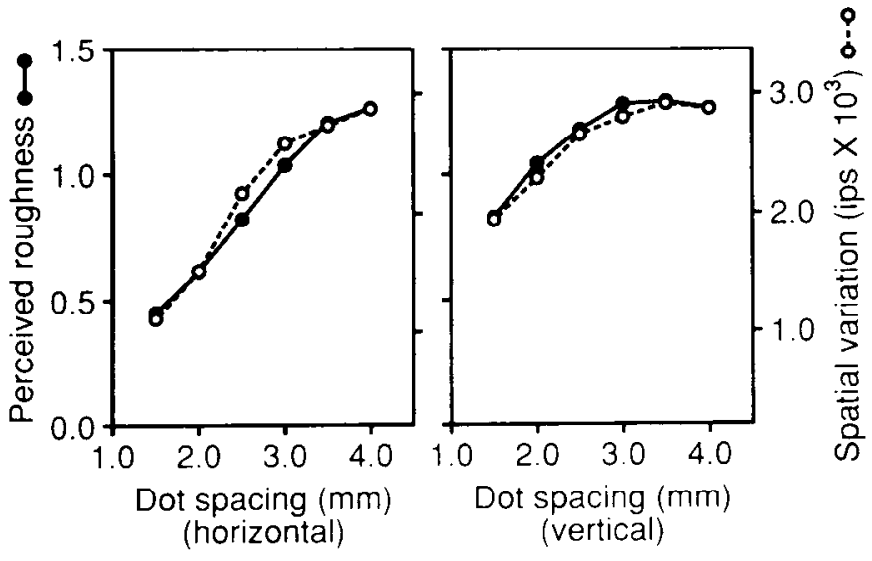

\section{B. RA SPATIAL VARIATION}

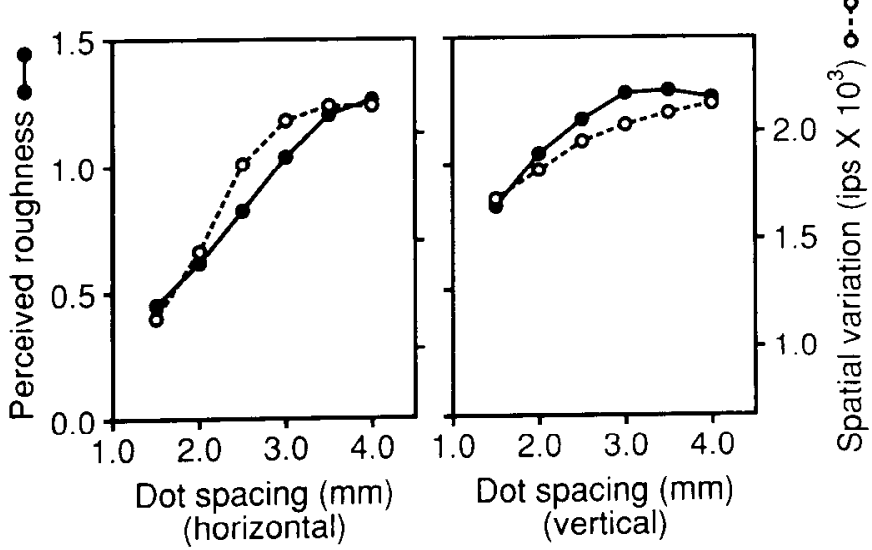

Figure 10. Spatial variation and roughness magnitude versus dot spacing, as in Figure 8 . $A$, SA spatial variation; average $\mathrm{SEM}=260$ ips; correlation coefficient $=0.984$. $B$, RA spatial variation; average SEM $=120$ ips; correlation coefficient $=0.938$.

RA spatial variation (i.e., values near 200 ips) produce very different sensations of roughness. These results support the hypothesis that roughness perception is based on between-afferent spatial variation in the activity of SA afferents. The comparable hypothesis for RA afferents cannot be rejected but is less compelling.

The effects of varying spatial period and filter size in the SA analysis are shown in Figure 12. Correlation was highest for a spatial period of $2.8 \mathrm{~mm}$, but remained above 0.9 over a range of $2.0-4.0 \mathrm{~mm}$. The best filter spread, given a spatial period of $2.8 \mathrm{~mm}$, corresponded to a Gaussian envelope of SD $1.12 \mathrm{~mm}$ (which covers about one cycle). Correlation staycd higher than 0.9 over a range of SDs from 0.84 to $2.24 \mathrm{~mm}$. The spatial Gabor filter shown in Figure 6 was constructed using the optimal parameters. This filter consists mainly of one large negative lobe and one large positive lobe separated by about $1.5 \mathrm{~mm}$. Such a filter is maximally responsive to a patch of low activity adjacent to a patch of high activity at a distance of 1-2 $\mathrm{mm}$. Thus, the analysis suggests that roughness sensations are based on local differences in firing rate between $\mathrm{SA}$ receptors separated by 1$2 \mathrm{~mm}$ on the skin surface.

Our previous analysis of spatial variation was based on filters consisting of just two points, one positive and one negative

\section{A. SA SPATIAL VARIATION}

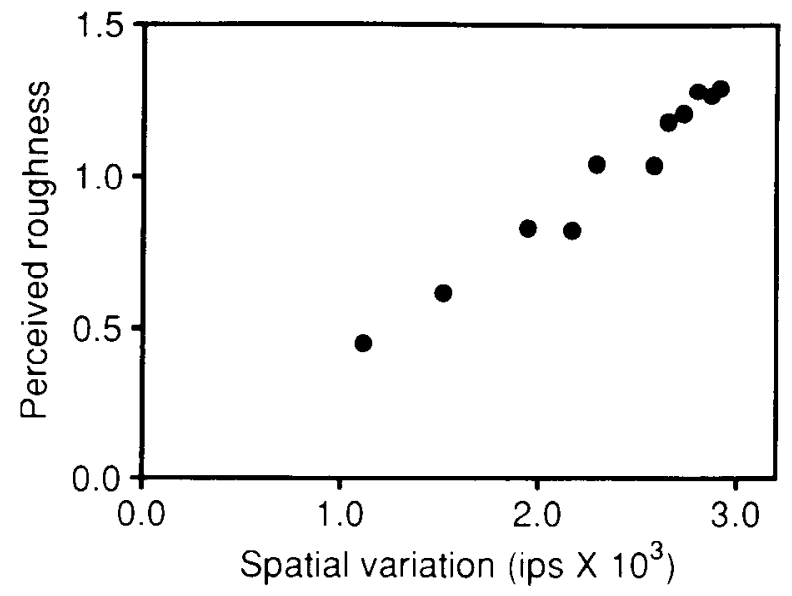

B. RA SPATIAL VARIATION

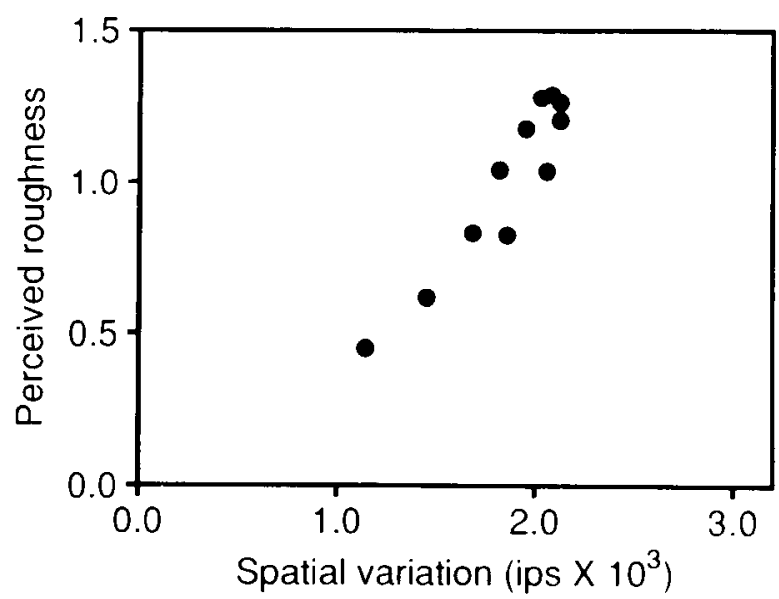

Figure 11. Roughness magnitude versus spatial variation. Each point corresponds to one of the 11 stimulus patterns. Position with respect to the $y$-axis indicates roughness magnitude for that pattern; position with respect to the $x$-axis indicates spatial variation for the same pattern. $A, \mathrm{SA}$ spatial variation. $B, \mathrm{RA}$ spatial variation.

(Connor et al., 1990). The two-point filters model the same type of mechanism as the Gabor filters, but are less selective for spatial frequency. This may explain the discrepancy in optimal spatial period between the two studies $(4.4 \mathrm{~mm}$ vs. $2.8 \mathrm{~mm})$. It was therefore of interest to apply the Gabor analysis to the older data. The older data set was based on 18 stimulus surfaces containing embossed dots arranged in a square array. The square arrays were oriented at $45^{\circ}$ relative to scanning direction so that the subjects scanned the arrays along the diagonal dimension. The spacing between dots (the sides of the squares) varied from 1.3 to $6.2 \mathrm{~mm}$; the diameter of the dots varied from $0.5 \mathrm{~mm}$ to $1.2 \mathrm{~mm}$. These surfaces were used in psychophysical experiments on 21 subjects and neurophysiological experiments on $11 \mathrm{SA}$ fibers. SEP data for the 11 SAs were analyzed using the optimal Gabor filters from the present study (period $-2.8 \mathrm{~mm}$, $\mathrm{SD}=1.12 \mathrm{~mm}$ ). The resulting values for spatial variation were closely related to roughness magnitude (correlation coefficient 
$=0.970$ ). The relationship between spatial variation and roughness magnitude across the entire set of 29 stimulus patterns is shown in Figure 13. The combined stimulus set produced a variety of effects on roughness due to dot size, dot spacing, and geometry relative to scanning direction. In all cases, the subjects' roughness judgments were correlated closely with spatial variation in SA impulse rate.

Spatial variation was also measured with DOG filters. The shape and spread of the DOG filters are determined by the SDs of the component 2-D Gaussians. The smaller SD was varied over a range of $0.1-1.2 \mathrm{~mm}$. The larger SD was varied from 1.25 to 4 times the smaller SD. The DOG filters were either "on-center," meaning the smaller Gaussian was positive and the larger negative, or "off-center," meaning the polarities were reversed. Polarity had almost no effect, so the results were averaged across the two conditions. The closest fit for the SA analysis was obtained with SDs of 0.6 and $0.9 \mathrm{~mm}$. These parameters produce the DOG filter shown in Figure 6. It is interesting that for this optimum DOG filter the distance between the center peak and the minimum point of the surround is 1.4 $\mathrm{mm}$, exactly the peak-to-trough distance for the optimal Gabor filters. Thus, the DOG analysis confirms the idea that roughness is based on spatial variations on a scale of $1-2 \mathrm{~mm}$. The correlation between psychophysical and neural data is much lower for the DOG results than for the Gabor results $(0.927$ vs. 0.984 , which corresponds to $14 \%$ unexplained variation vs. $3 \%$ ). The optimum correlation for the RA analysis was even lower $(0.824$ vs. $0.938 ; \mathrm{SD}=0.3$ and $0.9 \mathrm{~mm}$ ).

\section{Discussion}

The results presented here extend our previous findings concerning neural coding of tactile roughness (Connor et al., 1990) in three ways. First, they confirm that perceived roughness is not based on mean firing rate of peripheral mechanoreceptive fibers. Earlier results with square dot array stimuli indicated that roughness does not depend on mean rate in the SA, RA, or $\mathrm{PC}$ populations or on any combination of mean rate across populations. The experiments with linear dot arrays reported here revealed an even more striking lack of correlation between roughness and mean rate. It has also been demonstrated that the roughness of grating surfaces cannot be fully explained in terms of mean firing rate (Sathian et al., 1989). Thus, it has now been shown for a variety of stimuli that mean firing rate fails to account for perceived roughness.

Second, the results indicate that roughness is not based on within-afferent temporal variation in response rates. The previous study had shown a close correlation between roughness magnitude and temporal variation in SA (and to a lesser extent RA) firing rates. However, the critical period of temporal variation changcd with scanning vclocity. At a scanning vclocity of $20 \mathrm{~mm} / \mathrm{sec}$, variations on a scale of $130 \mathrm{msec}$ produced the closest correlation with roughness magnitude. At $50 \mathrm{~mm} / \mathrm{sec}$, the optimal scale was $44 \mathrm{msec}$. These two temporal intervals correspond to approximately the same distance of travel in space ( 2.2 and $2.6 \mathrm{~mm}$, respectively). Thus, the correlation between firing rate variation and roughness showed constancy for space rather than time. This led us to suspect that roughness sensations are actually based on spatial variation rather than temporal variation. In the present study, we addressed the issue directly by manipulating the amount of temporal variation produced by the stimulus surfaces. The stimuli were linear dot arrays in which element spacing could be independently varied in the temporal

\section{A. SPATIAL PERIOD}

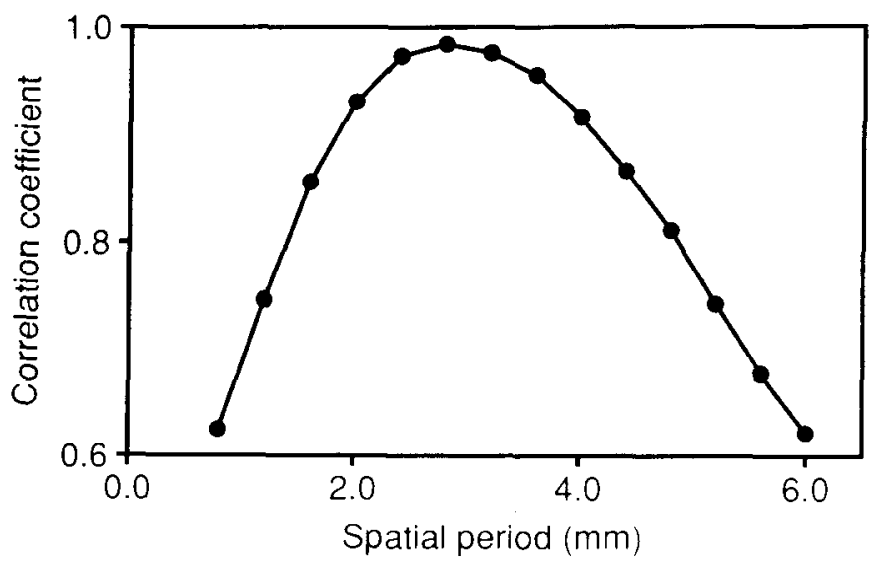

\section{B. STANDARD DEVIATION}

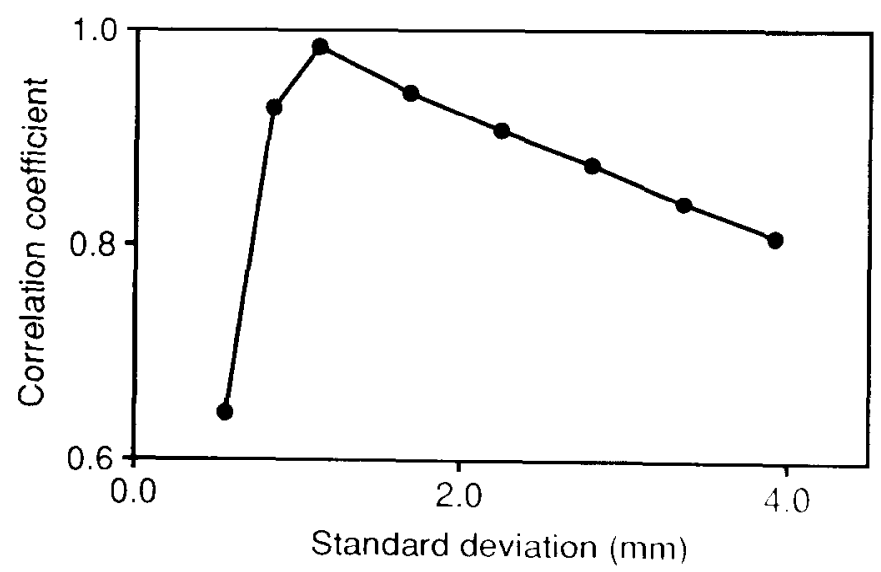

Figure 12. Correlation versus filter parameters. $A$, Correlation between $\mathrm{SA}$ spatial variation and roughness magnitude as a function of Gabor filter spatial period. For each point, $\sigma=0.4 \lambda . B$, Correlation between SA spatial variation and roughness magnitude as a function of Gabor filter size. For each point, $\lambda=2.8 \mathrm{~mm}$.

direction (the direction of scanning) and the nontemporal direction (perpendicular to scanning). It was found that, as element spacing increased in the nontemporal direction, temporal variation in SA and RA response rates decreased. Over the same range, however, roughness magnitude actually increased. This finding, in combination with the previous evidence, makes it clear that roughness sensations are not based on temporal variation in mechanoreceptor firing rate.

Third, the present results provide further evidence that roughness sensations are based on between-afferent spatial variation in firing rates of SA and possibly RA afferents. The correlation between spatial variation and roughness magnitude now appears to hold across a wide range of stimulus conditions. The previous study showed that, for the square array dot patterns, as dot spacing increased, roughness magnitude increased to a peak at about $3.0 \mathrm{~mm}$ and then declined at larger spacings. This behavior was duplicated by spatial variation (see Fig. 13). It was also found that roughness decreased at larger dot sizes; the same effect was seen for spatial variation. The correlation remained 


\section{SA SPATIAL VARIATION}

Figure 13. SA spatial variation and roughness magnitude for combined data sets, as in Figure 8. Upper panels, Present data set. Lower panels, Old data set (see Connor et al., 1990). Stimuli in the old set were 18 surfaces containing dots of $0.5,0.7$, or $1.2 \mathrm{~mm}$ diameter arranged in a square array at spacings of $1.3,2.4,3.2,4.3,5.2$, or $6.2 \mathrm{~mm}$. Roughness magnitude (open symbols) represents average normalized values for 21 subjects. Spatial variation (solid symbols) represents averages over 11 SA afferents. Scaling of the axes for the two data sets was necessarily based on separate regressions because the two sets of psychophysical data had to be normalized separately. After normalization, the value 1.0 represents the average roughness for each stimulus set, and presumably the two sets have different averages (and different ranges). It appears, based on the relative positions of the right-hand axes, that average roughness was higher for the new stimulus set: the value 1.0 for the new stimulus set corresponds to 2300 ips, while 1.0 for the old stimulus set corresponds to 1400 ips. This presumed difference in roughness conforms to our subjective impressions but has not been tested experimentally.
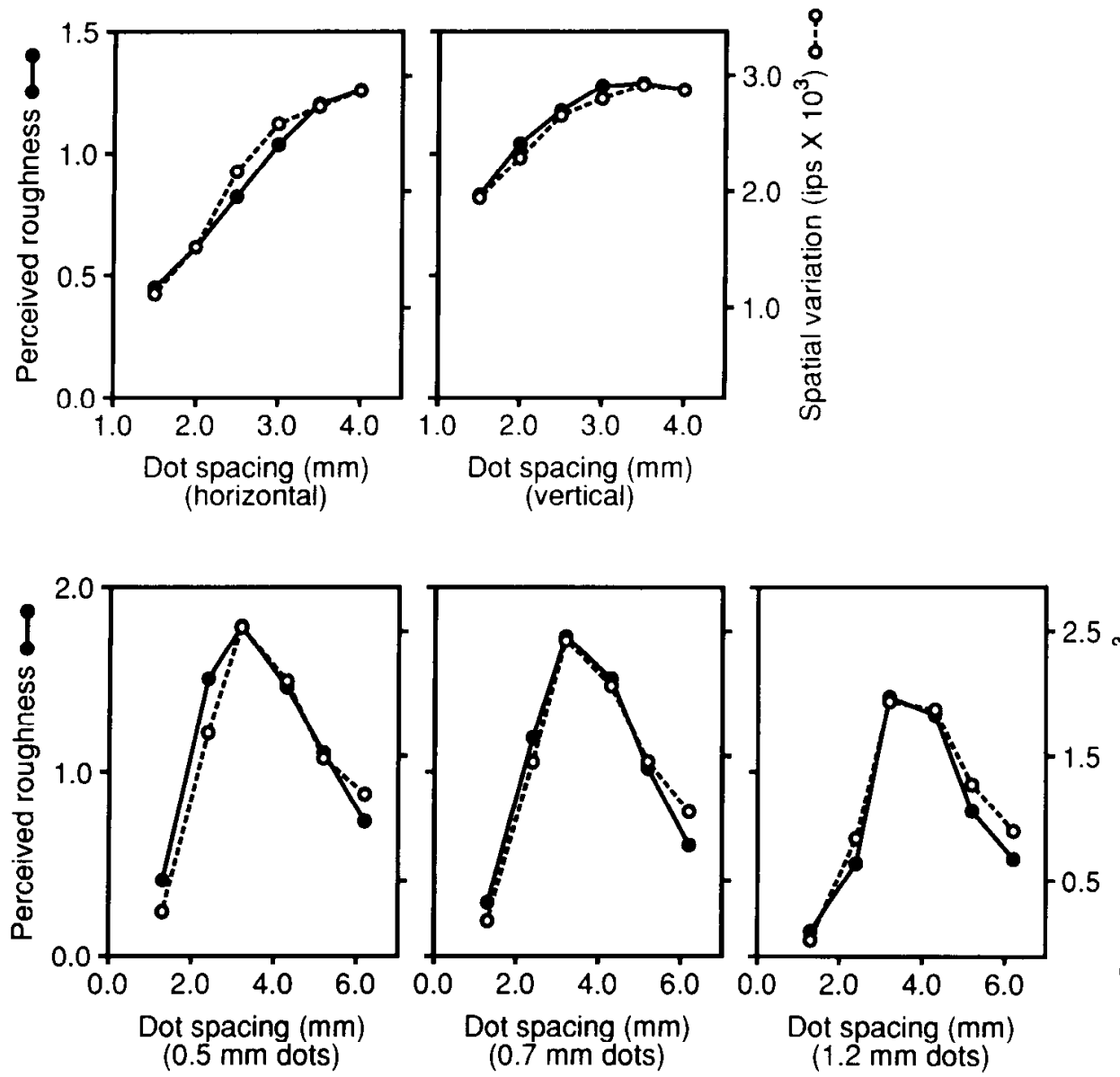

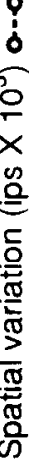

high for different scanning velocities $(20 \mathrm{~mm} / \mathrm{sec}$ and $50 \mathrm{~mm} /$ $\mathrm{sec}$ ). In the present study, for stimuli consisting of linear dot arrays, roughness magnitude declined at close dot spacings. The decline was sharper for close spacings in the scanning direction. Once again, this behavior was mirrored by spatial variation. In all cases, the correlation between roughness and spatial variation in SA responses was high $(>0.97)$. For the entire set of $29 \mathrm{dot}$ surfaces, spatial variation accurately predicts relative intensities of roughness sensations.

The exact nature of the spatial variations critical for roughness perception is uncertain, but a few generalizations can be made. First, the relevant variations are local. Correlation was highest for spatial filters encompassing about one cycle $(2.8 \mathrm{~mm}$ at the optimal spatial frequency) and declined significantly as more cycles were included (see Fig. 12). Thus, roughness does not depend on some measure of global variation akin to the Fourier power spectrum. Second, the relevant period of variation is in the range of $2-4 \mathrm{~mm}$ (see Fig. 12). In other words, roughness depends on differences in firing rate across distances of $1-2 \mathrm{~mm}$ on the skin surface. This corresponds to differences in firing rate between adjacent or nearly adjacent receptors (see Johansson and Vallbo, 1979; Darian-Smith and Kenins, 1980), that is, the finest spatial differences that the system can measure. Third, roughness appears most closely related to spatial variation in the SA population. The data do not allow an unequivocal differentiation between the roles of SA and RA afferents: there is a consistent relationship between roughness magnitude and spatial variation in both SA and RA firing rates. However, the relationship between roughness magnitude and SA spatial variation is proportional and highly consistent, whereas the RA relationship is not proportional and is less consistent (see Fig. 11). A linear regression describes the relationship between roughness magnitude and spatial variation for both the SA and RA afferents (as indicated by the correlation coefficients, 0.984 and 0.938 , respectively), but only the SA intercept lies near the origin. If RA spatial variation were advanced as the basis for roughness perception, then the relationship would have to include a very high threshold, that is, a very high value of spatial variation before any sensation of roughness is evoked. A special role for SAs in texture perception would conform with the notion that SAs are particularly adapted for spatial processing (scc Johnson and Hsiao, 1992).

The conclusions presented here may be valid only for stimuli with textural structure on a scale of millimeters. The finest surfaces used in this series had a dot spacing of $1.3 \mathrm{~mm}$. The spatial resolution limit of the peripheral somatosensory system (specifically the SA afferents) is approximately $0.5 \mathrm{~mm}$ (Phillips and Johnson, 1981). Textural elements of this size or greater produce a spatial pattern of activity across the receptor population that carries information about surface structure. It is reasonable that for textures of this kind roughness sensations would be based on a between-afferent spatial code. However, roughness is also 
a quality of surfaces with feature dimensions measured on a scale of tenths of millimeters or even microns. It is not clear that the mechanism proposed here would account for roughness perception for these finer surfaces.

Fine textures that fail to produce a clear spatial image still evoke vibrations to which the somatosensory system is extremely sensitive. Katz (1925) proposed that the vibratory sense might underlie the sense of roughness for very fine stimuli as well as stimuli explored through an intermediate object. PC afferents are extremely sensitive (thresholds less than $0.1 \mu \mathrm{m}$ ) to vibration in the $60-500 \mathrm{~Hz}$ range, RA afferents are very sensitive (thresholds down to $1 \mu \mathrm{m}$ ) in the $5-200 \mathrm{~Hz}$ range, and SA afferents are quite sensitive (thresholds to $10 \mu \mathrm{m}$ ) in the $0-100 \mathrm{~Hz}$ range (Mountcastle et al., 1972; Johnson, 1974; Freeman and Johnson, 1982). RA afferents can respond to texture elements as small as $2 \mu \mathrm{m}$ in height, and PC afferents can respond to elements just $0.1 \mu \mathrm{m}$ in height (Johansson and LaMotte, 1983; LaMotte and Whitehouse, 1986; LaMotte and Srinivasan, 1990; for further discussion, see Johnson and Hsiao, 1992).

If a new study were undertaken using finer surfaces, the rate, temporal, and spatial codes would have to be reconsidered. Johnson (1983) provided some evidence that the relative impulse rates of SA, RA, and PC afferents depend on spatial structure in finely texlured surfaces and that this might account for the human capacity to discriminate such surfaces. A temporal code might account for the roughness of fine surfaces, although it would suffer from a sensitivity to scanning rate regardless of the spatial structure of the stimulus. Spatial coding mechanisms of the kind considered here are not ruled out simply because the spatial detail is below the limits of spatial acuity. A very fine surface evokes population activity in which there is certain to be spatial variation between fibers. Any such variation will activate central mechanisms of the type considered in this article.

The idea that roughness corresponds to spatial variation in firing rates of peripheral mechanoreceptive fibers has implications for central mechanisms of texture processing. A primary function for central processing of somatosensory information must be form analysis (see, e.g., Johnson and Hsiao, 1992). Form analysis is necessary for accurately grasping, recognizing, and manipulating objects. Any system for form analysis must begin by extracting spatial differences in activity across the primary receptor sheet. This is accomplished by central neurons with receptive fields containing antagonistic excitatory and inhibitory subregions. In the visual system, spatial differences in activity are extracted by neurons with center-surround and banded receptive field structures. Comparable mechanisms must exist at some point in the somatosensory system (cf. Bankman et al., 1991). Such cells act just like the spatial filters used in the analysis here, responding to local variations in the activity of primary receptors. In fact, the response properties of centersurround and banded (simple) cells have been modeled with DOG and 2-D Gabor filters, respectively (see, e.g., Rodieck, 1965; Jones and Palmer, 1987). The average activity in a population of such cells is proportional to the overall amount of spatial variation in the peripheral image. It may be that roughness corresponds to average activity in a population of cells adapted for somatosensory form processing. At the peripheral level, roughness appears to depend on spatial variations on a scale of 1-2 $\mathrm{mm}$; at the cortical level, roughness may depend on the overall activity in a population of cells specifically sensitive to such variations. At present, we favor the hypothesis that tactile roughness perception is proportional to the mean firing rate in a population of central neurons sensitive to spatial variation in the activity of SA afferent fibers.

\section{References}

Bankman IN, Hsiao SS, Johnson KO (1991) Neural image transformation in the somatosensory system of the monkey: comparison of neurophysiological observations with responses in a neural network model. Cold Spring Harbor Symp Quant Biol 55:61 1-620.

Connor CE, Hsiao SS, Phillips JR, Johnson KO (1990) Tactile roughness: neural codes that account for psychophysical magnitude estimates. J Neurosci 10:3823-3836.

Darian-Smith I, Kenins P (1980) Innervation density of mechanoreceptive fibers supplying glabrous skin of the monkey's index finger. I Physiol (Lond) 309:147-155.

Daugman JG (1980) Two-dimensional spectral analysis of cortical receptive field profiles. Vision Res 20:847-856.

Daugman JG (1985) Uncertainty relation for resolution in space, spatial frequency, and orientation optimized by two-dimensional visual cortical filters. J Opt Soc Am 2:1160-1169.

Delgutte B (1987) Peripheral auditory processing of speech information: implications from a physiological study of intensity discrimination. In: The psychophysics of speech perception (Schouten MEH, ed), pp 333-353. Dordrecht: Martinus Nijhoff.

Freeman AW, Johnson KO (1982) A model accounting for effects of vibratory amplitude on responses of cutaneous mechanoreceptors in macaque monkey. J Physiol (Lond) 323:43-64.

Gabor D (1946) Theory of communication. J IEE (Lond) 93:429-457.

Heller MA (1982) Visual and tactual texture perception: intersensory cooperation. Percept Psychophys 31:339-344.

Johansson RS, LaMotte RH (1983) Tactile detection thresholds for a single asperity on an otherwise smooth surface. Somatosens Res 1: 21-31.

Johansson RS, Vallbo A (1979) Tactile sensitivity in the human hand: relative and absolute densities of four types of mechanoreceptive units in glabrous skin. J Physiol (Lond) 286:283-300.

Johnson KO (1974) Reconstruction of population response to a vibratory stimulus in quickly adapting mechanoreceptive afferent fiber populations innervating glabrous skin of the monkey. J Neurophysiol 37:48-72.

Johnson KO (1983) Neural mechanisms of tactual form and texture discrimination. Fed Proc 42:2542-2547.

Johnson KO, Hsiao SS (1992) Neural mechanisms of tactual form and texture perception. Annu Rev Neurosci 15:227-250.

Johnson KO, Lamb GD (1981) Neural mechanisms of spatial tactile discrimination: neural patterns evoked by Braille-like dot patterns in the monkey. J Physiol (Lond) 310:117-144.

Johnson KO, Phillips JR (1988) A rotating drum stimulator for scanning embossed patterns and textures across the skin. J Neurosci Methods $22: 221-231$.

Johnson KO, Darian-Smith I, LaMotte C (1973) Peripheral neural determinants of temperature discrimination in man: a correlative study of responses to cooling skin. J Neurophysiol 36:347-370.

Jones JP, Palmer LA (1987) An evaluation of the two-dimensional Gabor filter model of simple receptive fields in cat striate cortex. J Neurophysiol 58:1233-1258.

Katz D (1925) Der Aufbau der Tastwelt. Z Psychol Erganzungshand 11. Reprinted in The world of touch (Krueger LE, transl). Hillsdale, NJ: Erlbaum, 1989.

LaMottc RH (1977) Psychophysical and neurophysiological studies of tactile sensibility. In: Clothing comfort: interaction of thermal, ventilation construction and assessment factors (Hollies N, Goldman R, eds), pp 83-105. Ann Arbor, MI: Ann Arbor Science.

LaMotte RH, Srinivasan MA (1990) Surface microgeometry: neural encoding and perception. In: Information processing in the somatosensory system (Franzen $O$, Westman $J$, eds), pp 49-58. London: Macmillan.

LaMotte RH, Whitehouse J (1986) Tactile detection of a dot on a smooth surface: peripheral neural events. J Neurosci 6:1 109-1128.

Lederman SJ (1974) Tactile roughness of grooved surfaces: the touching process and effects of macro- and microsurface structure. Percept Psychophys 16:385-395.

Lederman SJ (1982) The perception of texture by touch. In: Tactual 
perception: a sourcebook (Schiff W, Foulke E, eds), pp 130-167. Cambridge: Cambridge UP.

Marcelja S (1980) Mathematical description of the responses of simple cortical cells. J Opt Soc Am 70:1297-1300.

Meenes M, Zigler MJ (1923) An experimental study of the perceptions of roughness and smoothness. Am J Psychol 34:542-549.

Mountcastle VB, LaMotte RH, Carli G (1972) Detection thresholds for stimuli in humans and monkeys: comparison with threshold events in mechanoreceptive afferent nerve fibers innervating the monkey hand. J Neurophysiol 35:122-136.

Phillips JR, Johnson KO (1981) Tactile spatial resolution. II. Neural representation of bars, edges, and gratings in monkey afferents. J Neurophysiol 46:1 192-1203.

Phillips JR, Johnson KO, Hsiao SS (1988) Spatial pattern representation and transformation in monkey somatosensory cortex. Proc Natl Acad Sci USA 85:1317-1321.

Rodieck RW (1965) Quantitative analysis of cat retinal ganglion cell response to visual stimuli. Vision Res 5:583-601.

Sathian K, Goodwin AW, John KT, Darian-Smith I (1989) Perceived roughness of a grating: correlation with responses of mechanorecep- tive afferents innervating the monkey's finger pad. J Neurosci 9:12731279.

Stevens SS, Harris J (1962) Scaling of roughness and smoothness. J Exp Psychol 64:489-494.

Talbot WH, Darian-Smith I, Kornhuber HH, Mountcastle VB (1968) The sense of flutter-vibration: comparison of the human capacity with response patterns of mechanoreceptive afferents from the monkey hand. J Neurophysiol 31:301-334.

Tamura H, Mori S, Yamawaki T (1978) Textural features corresponding to visual perception. IEEE Trans Syst Man Cybern 8:460-473.

Wendahl RW (1966) Some parameters of auditory roughness. Folia Phoniatr (Basel) 18:26-32.

Winslow RL, Sachs MB (1988) Single-tone intensity discrimination based on auditory-nerve rate responses in backgrounds of quiet, noise and with stimulation of the crossed olivocochlear bundle. Hearing Res 35:165-190.

Worgotter F, Holt G (1991) Spatiotemporal mechanisms in receptive fields of visual cortical simple cells: a model. J Neurophysiol 65:494 510 . 